\title{
Occam's Razor or Hickam's Dictum: A Paraneoplastic or Coincidental Occurrence of Lung Cancer and Guillain-Barré Syndrome
}

\author{
Satoshi Watanuki ${ }^{1,2}$, Kensuke Kinoshita ${ }^{1}$, Akiko Oda ${ }^{1}$, Hiroyuki Kobayashi ${ }^{1}$, \\ Hiroaki Satoh ${ }^{1}$ and Yasuharu Tokuda ${ }^{1}$
}

\begin{abstract}
A 67-year-old man was admitted due to weakness, coughing, shortness of breath and fever. He had decreased breath sounds in the left lung and muscle weakness in the lower and upper extremities. Chest imaging showed a mass in the left lung, and a biopsy revealed small cell lung cancer. The nerve conduction velocity was decreased, and anti-GM1 IgG antibodies were positive. The patient showed a temporary neurologic recovery following the administration of cancer chemotherapy, although he eventually died of progression of lung cancer. As a result of the almost simultaneous symptomatic development of lung cancer and Guillain-Barré syndrome, this case may be considered to involve a paraneoplastic neurologic syndrome.
\end{abstract}

Key words: Guillain-Barré syndrome, paraneoplastic syndrome, lung cancer

(Intern Med 53: 1569-1573, 2014)

(DOI: 10.2169/internalmedicine.53.2098)

\section{Introduction}

Peripheral neuropathy is commonly accompanied by cancer, while the isolated coincidence of immune-mediated neuropathy and cancer in a single patient is rare. We herein report a case of lung cancer in a patient who developed immune-mediated neuropathy with positive GM1ganglioside autoantibodies. In addition, we provide a discussion of etiologic considerations based on the views of clinical reasoning.

\section{Case Report}

A 67-year-old man presented with a four-week history of a productive cough, shortness of breath and fever up to 38 degrees Celsius. Antibiotics were prescribed by his home doctor for suspected pneumonia without any improvement. Because he developed bilateral leg weakness and was unable to walk for two days, he was transferred and admitted to our hospital. His past medical history included schizophrenia starting at 17 years of age, and his current medications included chlorpromazine, promethazine, phenobarbital and haloperidol. He denied allergies, exposure to animals or recent travel. He lived in a wooden house and worked as a helper for a vocational training facility for the mentally disabled. He drank a moderate amount of alcohol and had smoked one pack of cigarettes per day for the last 40 years. A review of the systems revealed anorexia and significant weight loss of approximately 10 kilograms within the last eight months. No diarrhea, rashes or muscle or joint pain were observed.

On a physical examination, the patient was alert and oriented, although he appeared chronically ill. His temperature was 37.0 degrees Celsius, his pulse was 87 beats per minute, his blood pressure was $116 / 70 \mathrm{mmHg}$, his respiratory rate was 24 breaths per minute and his pulse oximetry oxygen saturation was $96 \%$ while breathing ambient air. There were decreased breath sounds in the left lung in addition to finger clubbing. No superficial lymphadenopathy was identified.

On a neurological examination, the patient exhibited nor-

${ }^{1}$ Department of Medicine, Mito Kyodo General Hospital, University of Tsukuba, Japan and ${ }^{2}$ Department of Rheumatology, Tokyo Metropolitan Tama Medical Center, Japan

Received for publication November 12, 2013; Accepted for publication January 19, 2014

Correspondence to Dr. Yasuharu Tokuda, yasuharu.tokuda@gmail.com 
mal visual fields, extraocular movement, size and reflexes of the pupils and facial sensory and motor functions. Sensory exams showed normal findings for pinprick, temperature, positional and vibrational sensations. Manual muscle testing revealed distal dominant mild muscle weakness of the bilateral upper extremities with distal dominant moderate to severe muscle weakness of the bilateral lower extremities. On the deep tendon reflex examination, there were diminished reflexes in the upper and lower extremities. No pathological reflexes were observed.

In laboratory data, a complete blood count showed a leukocyte level of $6,600 / \mu \mathrm{L}$, a hemoglobin level of $12.0 \mathrm{~g} / \mathrm{dL}$ and a platelet count of $316,000 / \mu \mathrm{L}$. Meanwhile, blood chemistry showed normal levels of electrolytes, glucose, creatinine phosphokinase, liver and renal function parameters, although the serum concentration of albumin $(2.4 \mathrm{~g} / \mathrm{dL})$ was decreased. An arterial blood gas analysis on ambient air showed a $\mathrm{pH}$ of 7.428, a $\mathrm{pCO}_{2}$ of $45 \mathrm{mmHg}$, a $\mathrm{pO}_{2}$ of 60 $\mathrm{mmHg}$ and $\mathrm{HCO}_{3}$ of $29 \mathrm{mEq} / \mathrm{L}$. The serum concentrations of vitamins B1 and B12 were normal, as were the results of

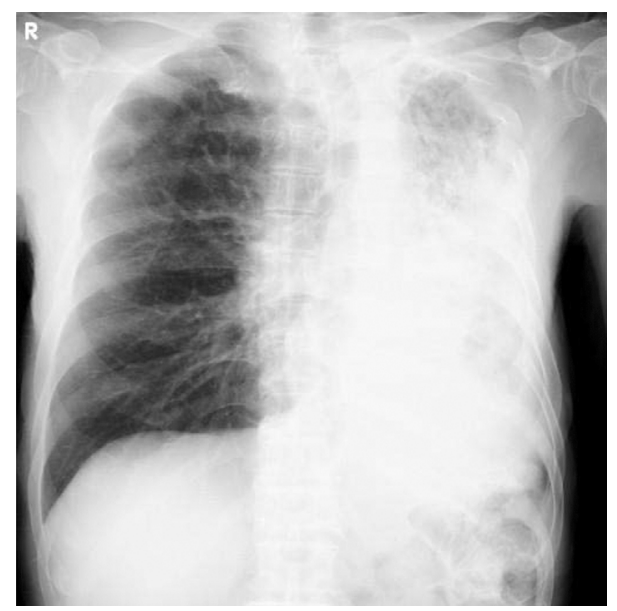

Figure 1. Chest X-ray: There is a shadow in the left lung field. an electrocardiogram. A chest X-ray film and CT scan showed a mass lesion associated with atelectasis of the left lung (Fig. 1, 2). A lumbar puncture was performed, which revealed an opening pressure of $70 \mathrm{mmH}_{2} \mathrm{O}$, a cell count of $0 / \mu \mathrm{L}$, a protein level of $25 \mathrm{mg} / \mathrm{dL}$ and a glucose level of 74 $\mathrm{mg} / \mathrm{dL}$ (serum glucose: $101 \mathrm{mg} / \mathrm{dL}$ at the time of puncture). The findings of brain and spine MRI scans were normal (Fig. 3, 4).

Two pathological processes were considered in this case, including pulmonary and neurological abnormalities. In the differential diagnosis, we considered the possibility of lung cancer with paraneoplastic neuropathy or the coincidental occurrence of lung cancer and a form of polyneuropathy, such as Guillain-Barré syndrome (GBS). To evaluate the left lung illness, bronchoscopy was performed, which identified a mass lesion in the left main bronchus (Fig. 5). Meanwhile, a biopsy revealed small cell lung carcinoma. Investigations of the patient's neurological illness showed that the nerve conduction velocity was decreased in the bilateral tibial nerves (Fig. 6), while a repetitive stimulation test of the peripheral nerve was negative (Fig. 7). Anti-GM1 IgG antibodies were positive, although anti-neuronal antibodies, including $\mathrm{Ri}, \mathrm{Hu}$ and $\mathrm{Yo}$, were negative.

Since a diagnosis of immune-mediated neuropathy was considered, on day 2 , the patient received large-dose intravenous immunoglobulins, without any significant improvements in his muscle weakness. Starting on day 20, two courses of chemotherapy comprising carboplatin plus etoposide were initiated for small cell lung cancer. Following the administration of chemotherapy, a follow-up lung image showed tumor shrinkage, indicating a partial response. Fig. 8 shows the serial changes in the patient's manual muscle test scores during his hospital stay. He exhibited a temporary improvement in weakness after a course of cancer chemotherapy. However, thereafter, his weakness again worsened, and he became completely bedridden. He was unable to walk during the entire period of hospitalization and eventually died due to progression of lung cancer. An (a)

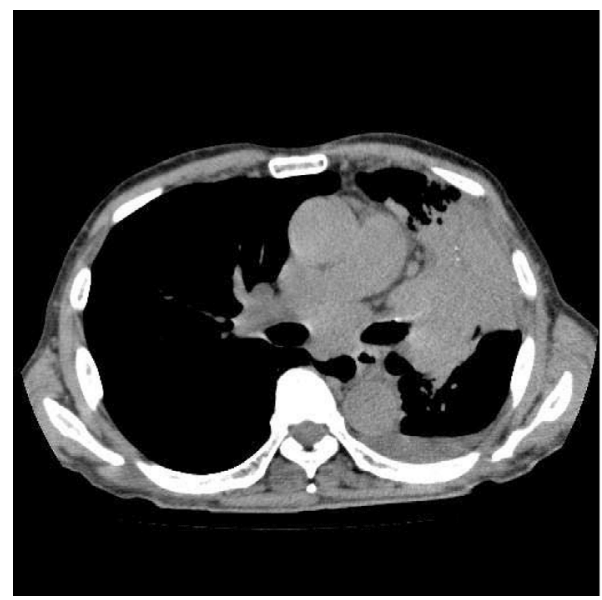

(b)

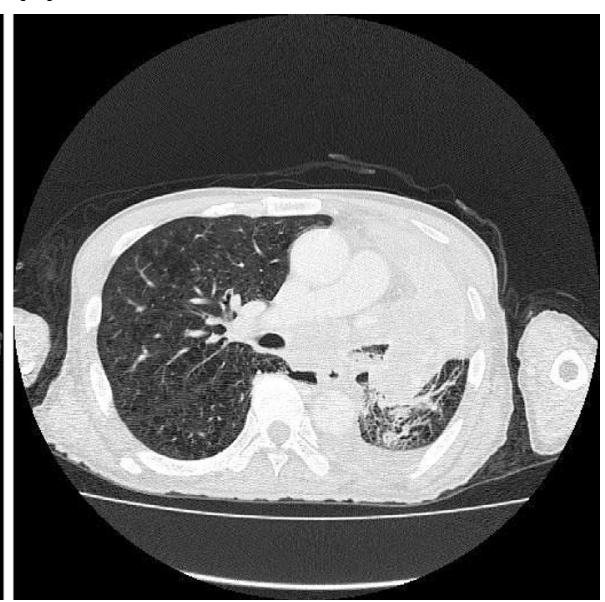

Figure 2. Chest CT scan: (a, b) There is a mass lesion in the left lung field. 


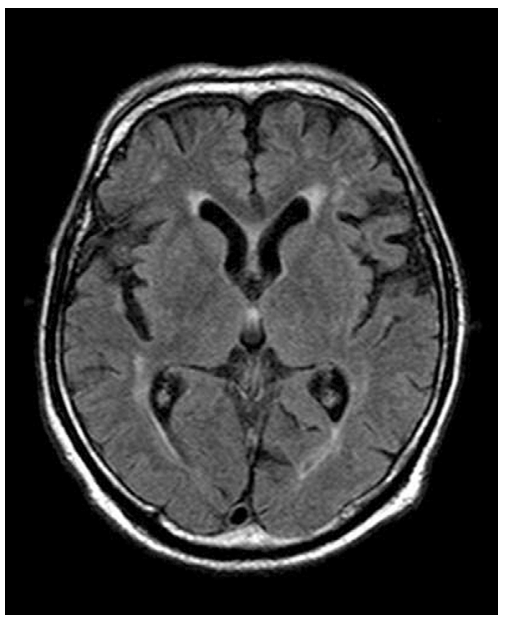

Figure 3. Brain MRI scan: No abnormalities

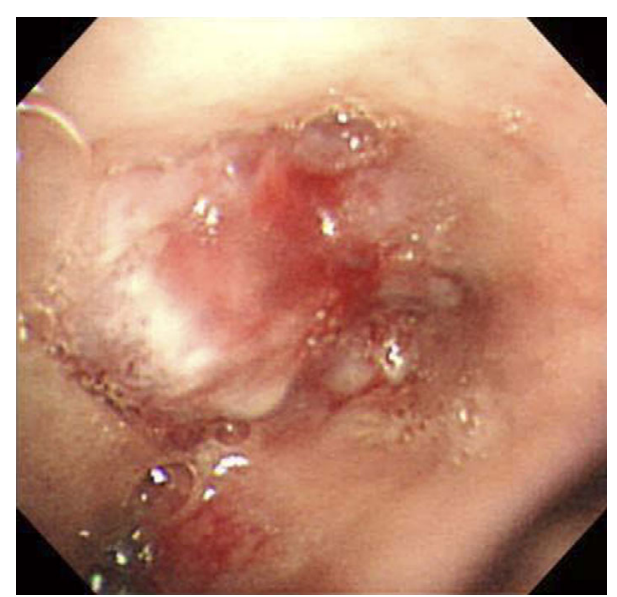

Figure 5. Bronchoscopic findings of the left main bronchus
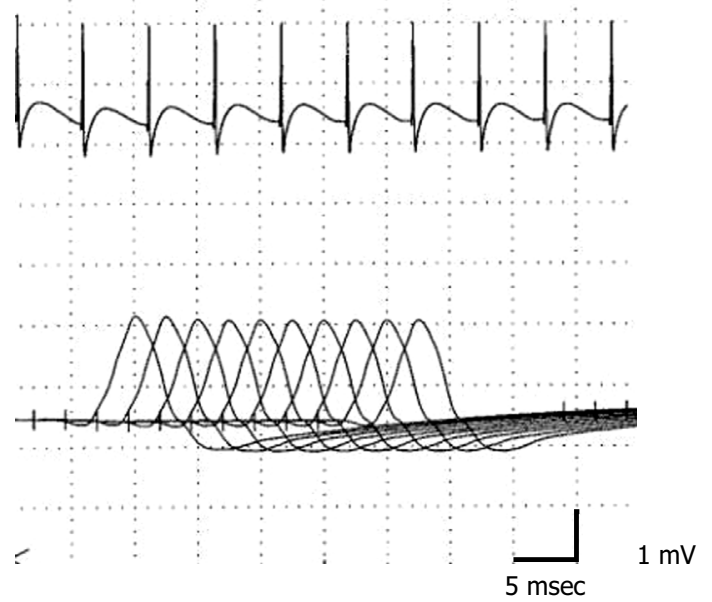

Figure 7. Repetitive stimulation test of the peripheral nerves: No waxing or waning phenomena were noted.

autopsy was not performed.

\section{Discussion}

GBS is an acute monophasic immune-mediated paralyzing

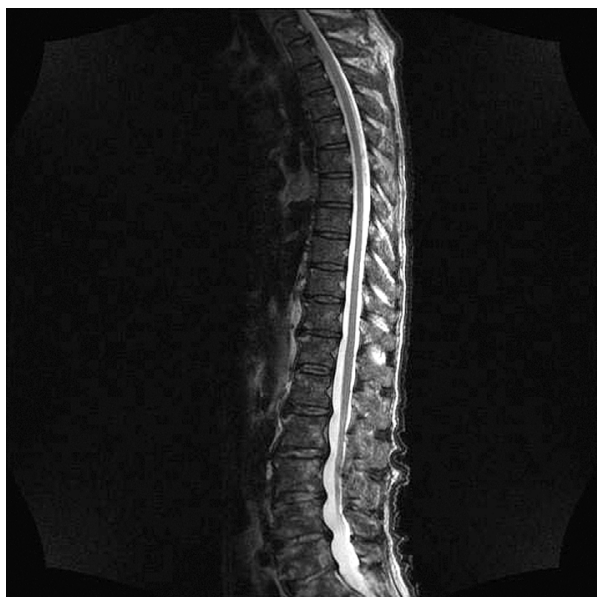

Figure 4. Spine MRI scan: No abnormalities

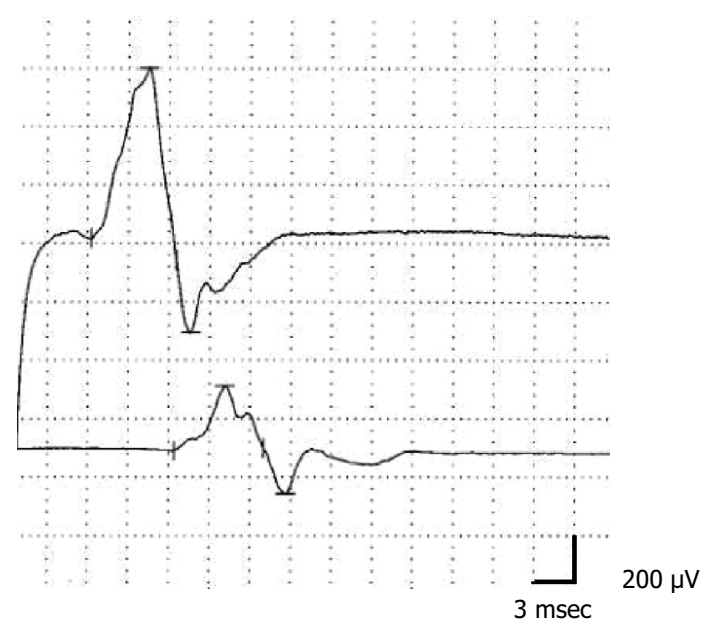

Figure 6. Nerve conduction velocity testing: A decreased nerve conduction velocity was observed in the bilateral tibial nerves.

polyneuropathy that is usually provoked by a preceding infection, such as Campylobacter jejuni enteritis, and it is now recognized to be a heterogeneous syndrome with several variant forms, including acute inflammatory demyelinating polyradiculoneuropathy, Miller Fisher syndrome, acute motor axonal neuropathy and acute sensorimotor axonal neuropathy (1). The mechanism underlying the development of GBS is considered to involve an immune response comprising molecular mimicry to the antigens of a preceding infection, stimulating a cross-reaction with peripheral nerve components.

Our patient exhibited a positive titer for IgG autoantibodies against GM1 ganglioside, an important component of peripheral nerves. IgG autoantibodies to GM1 ganglioside (ceramide attached to hexoses and monosialic acid linked to an oligosaccharide core) can be identified in patients with GBS (1). However, the median incidence of GBS is approximately one case per 100,000 person-years, thus indicating the rarity of the illness (2). On the other hand, paraneoplastic neuropathy is a relatively common complication of 


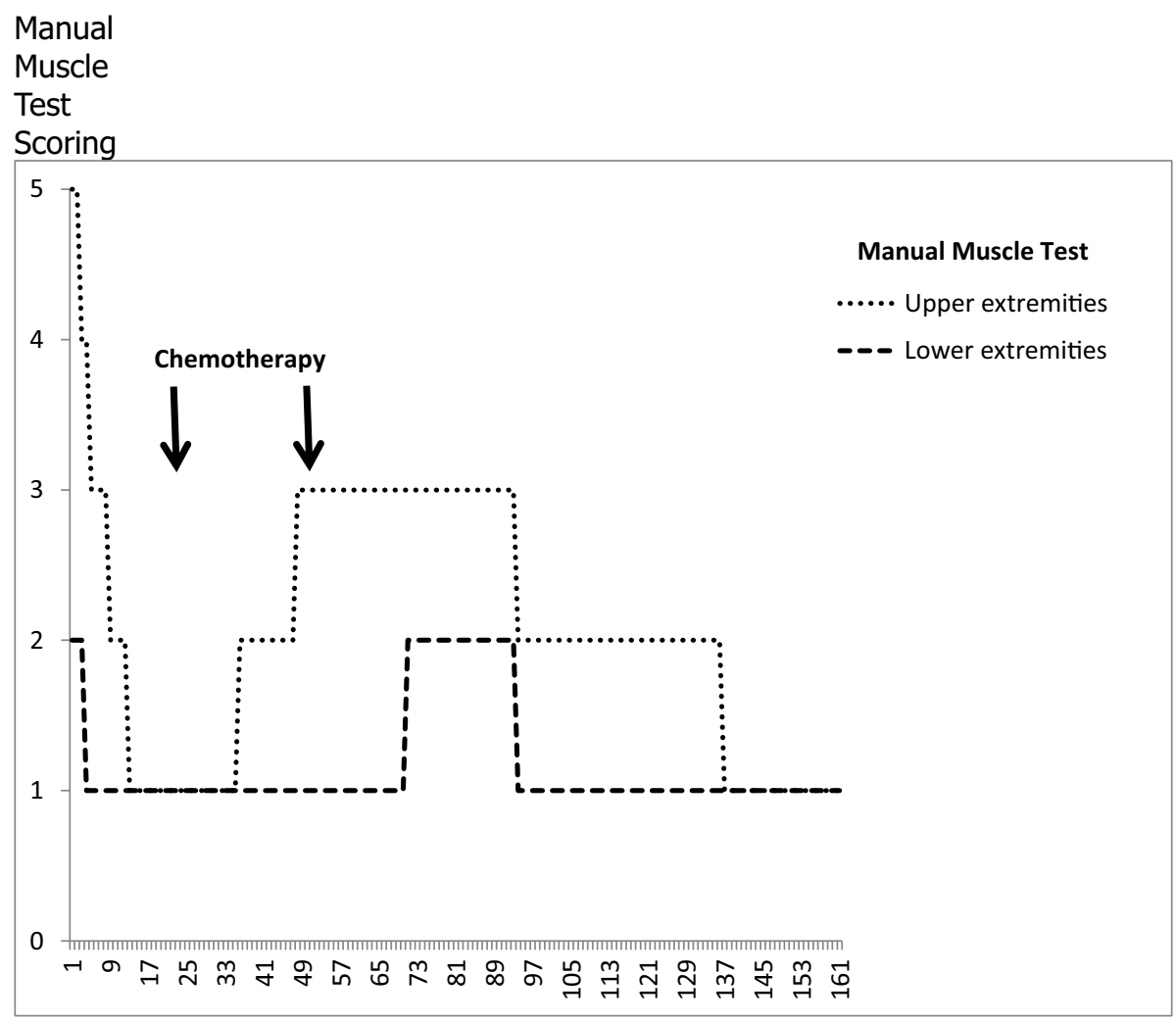

Days after admission

Figure 8. Serial changes in the patient's manual muscle test scores during his hospital stay

cancer, estimated to occur in approximately $1 \%$ of patients with malignancy (3). The development of GBS is occasionally associated with recent infections, such as that involving Campylobacter jejuni enteritis. Since the present patient had experienced a prior episode of fever, which may have been related to lung carcinoma, such as obstructive pneumonia, the GBS might have been associated with this preceding infection.

Several recent case reports have documented an association between GBS and malignancy $(4,5)$. Another previous report (6) described a case of paraneoplastic motor neuropathy in a patient with a high titer of anti-GM1 ganglioside antibodies associated with esophageal cancer. One study also indicated that the detection of gangliosides represents the presence of onconeural antigens in patients with paraneoplastic neuropathy and that the expression of gangliosides in neoplastic tissue can elicit autoimmune responses against neural structures (7). Therefore, the presence of GM1 autoantibodies should not be used to rule out a possible paraneoplastic etiology. The present patient exhibited a temporary improvement in weakness following the administration of chemotherapy for lung cancer. Therefore, the tumor shrinkage observed in this case may have resulted in a decreased expression of onconeural antigens as a target for an autoimmune response.

Several authors have proposed defining paraneoplastic neurological disorders according to the following items: the presence of a classical paraneoplastic neurological disorder, the detection of onconeural antibodies and the development of cancer within five years of the onset of a neurologic illness (8). Therefore, despite the absence of onconeural antibodies and classical neurologic features in the present case, the almost simultaneous symptomatic development of lung cancer and neuropathy should still be considered an association between lung cancer and neuropathy.

Clinical reasoning can help physicians to make an accurate diagnosis in complex cases. Based on Hickam's dictum, which states that, "a patient can have as many diagnoses as he darn well pleases" (9), more than one disease may be responsible for a patient's clinical signs and symptoms. On the other hand, however, William of Occam in the 14th century suggested that "plurality must not be posited without necessity" (9). A subsequent version of this statement was expressed as "among competing hypotheses, favor the simplest," hence the term "Occam's razor" (9). Since either principle cannot be embraced exclusively, we should honor the views of both Occam and Hickam and apply one of both appropriately to a particular set of complex manifestations. A study of diagnostic error showed that physicians occasionally rely on a single test result solely in order to make a diagnosis and may subsequently be trapped by the pitfall of base rate neglect, which may lead to misdiagnosis (10). When encountering cases involving rare diseases, such as GBS, in patients with a special disease state, such as cancer, physicians should embrace the parsimony of a diagnosis of paraneoplastic disease to explain such a combination, based 
on Occam's razor.

In conclusion, we herein reported the case of a 67-yearold man who concomitantly developed lung cancer and motor neuropathy with GM1 autoantibodies, suggesting a paraneoplastic GBS. In addition to the present case, several recent reports have also documented an association between GBS and malignancy. Therefore, the findings of anti-GM1 IgG autoantibody positivity should not be used to rule out a paraneoplastic etiology of peripheral neuropathy. Occam's razor can be applied as a diagnostic principle, as two simultaneous and special disease entities may in fact be considered a related condition.

The authors state that they have no Conflict of Interest (COI).

\section{References}

1. Yuki N, Hartung H-P. Guillain-Barré syndrome. N Engl J Med 366: 2294-2304, 2012.

2. Sejvar JJ, Baughman AL, Wise M, Morgan OW. Population incidence of Guillain-Barré syndrome: a systematic review and meta- analysis. Neuroepidemiology 36: 123-133, 2011.

3. Antoine JC, Camdessanche JP. Peripheral nervous system involvement in patients with cancer. Lancet Neurol 6: 75-86, 2007.

4. Naveed S, Okoli K, Hollingsworth J, Kasmani R. Guillain-Barré syndrome as a paraneoplastic manifestation of small-cell carcinoma of lung. South Med J 103: 156-158, 2010.

5. Togashi K, Shinohara H, Wakabayashi T, Fujita S, Sato K. Tetraplegia and respiratory failure due to carcinomatous neuropathy in the early postoperative period of a lung cancer patient: report of a case. Kyobu Geka (The Japanese Journal of Thoracic Surgery) 58: 495-498, 2005 (in Japanese).

6. Mostoufizadeh S, Souri M, de Seze J. A case of paraneoplastic demyelinating motor polyneuropathy. Case Rep Neurol 4: 71-76, 2012.

7. De Toni L, Marconi S, Nardelli E, et al. Gangliosides act as onconeural antigens in paraneoplastic neuropathies. J Neuroimmunol 156: 178-187, 2004.

8. Graus F, Delattre JY, Antoine JC, et al. Recommended diagnostic criteria for paraneoplastic neurological syndromes. J Neurol Neurosurg Psychiatry 75: 1135-1140, 2004.

9. Hilliard AA, Weinberger SE, Tierney LM Jr, Midthun DE, Saint S. Occam's razor versus Saint's triad. New England Journal of Medicine 350: 599-603, 2004.

10. Reilly JB, Ogdie AR, Von Feldt JM, Myers JS. Teaching about how doctors think: a longitudinal curriculum in cognitive bias and diagnostic error for residents. BMJ Qual Saf 22: 1044-1050, 2013.

(C) 2014 The Japanese Society of Internal Medicine http://www.naika.or.jp/imonline/index.html 